Europhysics Letters

PREPRINT

\title{
How short-ranged electrostatics controls the chromatin structure on much larger scales
}

\author{
Helmut Schiessel \\ Max-Planck-Institute for Polymer Research, Theory Group, POBox 3148, D-55021 Mainz, \\ Germany
}

PACS. 87.15.-v - Biomolecules: structure and physical properties.

PACS. 36.20.Ey - Conformation (statistics and dynamics.

\begin{abstract}
We propose that the degree of sweeling of the 30nm chromatin fiber (a "measure" of its transcriptional activity) is mainly determined by the short-ranged electrostatical interaction between different sections of the "folded" DNA chain. These sections constitute only a small fraction of the chain and they are located close to the entry-exit points of the DNA chain at the nucleosome core particles. We present a model that allows to estimate the degree of swelling of chromatin fibers as a function of salt concentration, charge density of the strands etc. Different mechanisms by which the state of chromatin can be controlled in vitro and in vivo are discussed.
\end{abstract}

DNA in eucaryotic cells is organized within a protein-DNA complex known as chromatin. In this way plant and animal genomes are compactified into volumes whose linear dimensions are many orders of magnitude smaller than their contour lengths. For instance, the human genom is made up of billions of base pairs (bp) corresponding to about one meter of DNA chains. These highly-charged and hard-to-bend polymers are condensed into complexes that have a characteristic size of a micron and therefore fit into the cell nucleus. At the same time it is of vital importance that a fraction of the genetic code stored within these tight complexes is accessible: For instance, gene regulatory proteins bind to specific sequences, the rather bulky protein complex RNA polymerase needs to gain access to a whole gene during its transcription etc. How DNA is "folded" within chromatin and how it can be "unfolded" for, say, transcription purposes is still poorly understood.

The primary structure of chromatin is known in great detail from x-ray studies [1]. The basic unit is the nucleosome consisting of the core particle and the linker DNA (of typical length $60 \mathrm{bp}$ ) that connects to the neighboring core particle. The resulting structure is a beads-on-chain necklace (10nm fiber). The core particle consists of $147 \mathrm{bp}$ DNA wrapped in 1- and 3/4 left-helical turns around a globular octamer of cationic proteins (core histones) forming a squat cylinder with a radius of about $5 \mathrm{~nm}$ and height of about $6 \mathrm{~nm}$. The higherorder secondary and tertiary structures on scales from $10 \mathrm{~nm}$ up to a micron are still a matter of controversy [2]. It is well-known that the bead-on-a-chain folds into a thicker fiber with a diameter of roughly $30 \mathrm{~nm}$ but it is still not clear how the neighboring beads are arranged in this fiber with respect to each other and where the linker is located. In the solenoid

(C) EDP Sciences 
models [3] it is assumed that the chain of nucleosomes forms a helical structure with the linker DNA being bent whereas the zig-zag- or crossed-linker models [4] posits straight linkers that connect core particles that are located at opposite sites of the fiber. An example of that kind of structure, the two-dimensional zig-zag fiber, is shown in Fig. 1(a). In general, the fiber is three-dimensional.

The difficulties in determining the structure of the $30 \mathrm{~nm}$ fibers are due to the lack of reliable experimental methods. Electron cryomicroscopy allows to visulize fibers in vitro but the fiber geometry can only be detected at ionic strengths well beyond physiological conditions where the structures are much more open 5]. Fibers at such low ionic strengths show straight linkers, a fact that supports the second class of the above mentioned chromatin models - at least for fibers at these unphysiological conditions. At higher ionic strengths, however, the fiber is so dense that their internal structure remains obscure.

A different approach allows to obtain structural information via an indirect method: the stretching of chromatin fibers with the help of micromanipulation devices as it was achieved recently. Cui and Bustamante [6] found that chromatin fibers are extremely soft with respect to their extension (compared to naked DNA). In principle, it should be possible to compare the obtained experimental data (namely the force-extension curves) with the existing models and to determine which is the appropriate model. In the meantime the crossed-linker model was indeed reevaluated in view of the stretching experiment. Katritch et al. [7] performed computer simulations showing that this model is capable of reproducing experimental data quite well for several sets of values of the "free" parameters. Schiessel et al. [8] gave an analytical treatment and introduced optimization criteria (see below) that led to reasonable predictions of the mechanical properties of the chromatin fiber without the use of any adjustable parameters. These studies do not invalidate the solenoid model but demonstrate at least that the crossed-linker model is successful in describing some characteristic features of the $30 \mathrm{~nm}$ fiber.

The main idea of the crossed-linker model as it was introduced by Woodcock and coworkers [4] is that the three-dimensional structure of the $30 \mathrm{~nm}$ chromatin fiber is determined by two quantities only: the entry-exit angle $\alpha$ of the linker DNA at the core particle $(c f$. Fig. 1(a)) and the linker length $b$. The entry-exit angle $\alpha$ is determined by local properties of the nucleosome core particle and it seems reasonable to assume that this quantity is constant throughout the fiber as long as there are homogeneous conditions. The second quantity, the linker length $b$, determines the rotational setting of neighboring nucleosomes. This follows from the fact that there are specific binding sites on the histone octamer where the DNA adsorbes with its minor groove facing inwards. Taking into account that the helical repeat length is $\approx 10 \mathrm{bp}$ it is clear that increasing $b$ by one bp leads to rotation of a nucleosome by $\approx 36^{\circ}$ with respect to its neighbor. As a consequence, changing $b$ by multiples of $\approx 10 \mathrm{bp}$ does not affect the rotational setting, i.e., the dihedral angle $\phi$ is periodic function of the linker length.

It was found that chromatin fibers have the tendency to show a preferential quantization with respect of their linker length and that this quantization is set by the helical twist 9]. This indicates that the dihedral angle $\phi$ shows a preferential value and thus the relative rotational setting is more or less constant throughout the fiber. Therefore it seems to be a reasonable approximation to consider both, $\alpha$ and $\phi$ to be constant throughout the fiber (together with $b$ ). The geometrical properties of the fiber, i.e., its radius $R$ and the linear density $\Lambda$ of nucleosomes along the fiber, are then a function of the two angles $\alpha$ and $\phi$ only (and of $b$ in a trivial sense). In Ref. [8] we termed this geometrical model therefore the two-angle model. A specific example of such a two-angle fiber with $\phi=180$ is the zig-zag fiber that we depicted in Fig. 1(a). 

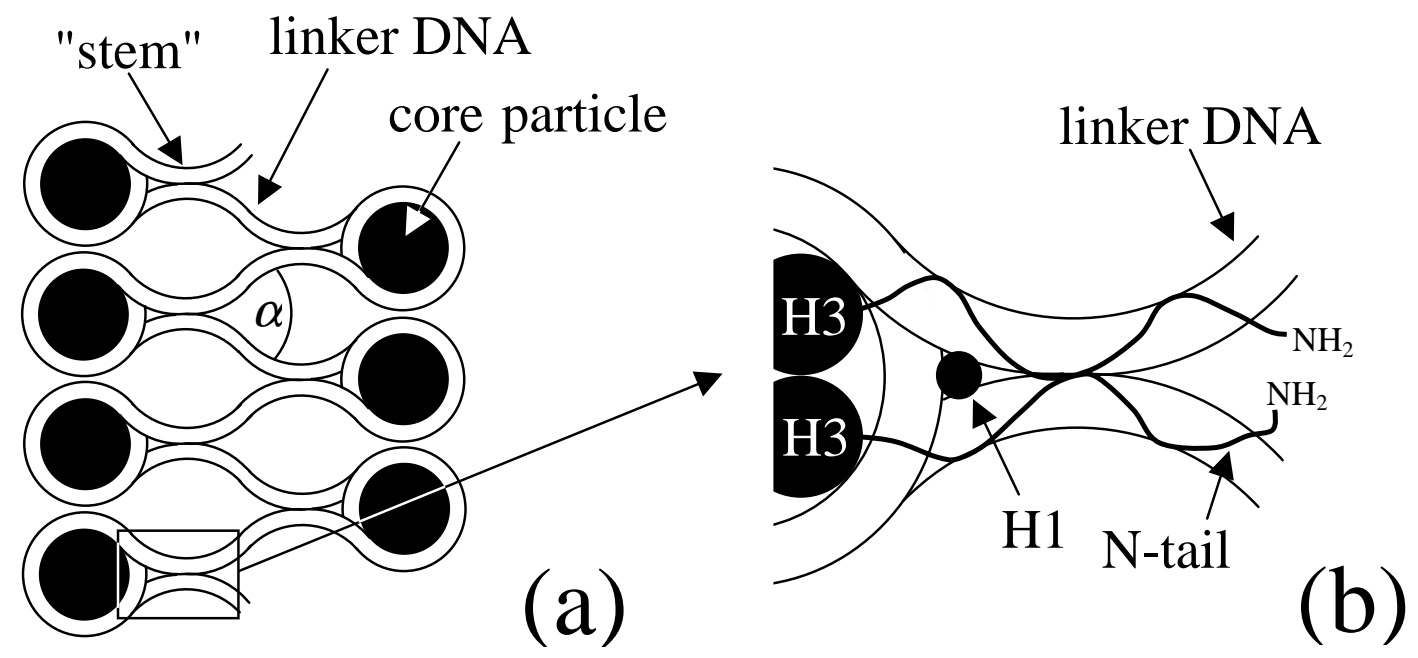

Fig. 1 - (a) Schematic view of a section of the 30nm chromatin fiber. For simplicity, the fiber is shown as a two-dimensional zig-zag fiber. The histone octamers are displayed as black circular disks. The DNA (white) consists of wrapped parts, "stem" sections and sections that link neighboring nucleosomes. (b) Enlarged view of the stem region. As shown schematically the stem section of the DNA interacts with cationic N-tails of the core histones and with the linker histone H1 (see text for details).

In our study of the geometrical and mechanical properties of the two-angle model [8] we suggested that the values of $\alpha$ and $\phi$ of the "native" 30nm chromatin fiber in its "silenced" form (no transcriptional activity) are chosen in such a way that the three-dimensional density of the fiber (defined by $\Lambda / \pi R^{2}$ ) and its "accessibility" are optimized. Accessibility means here how rapid the "silenced" fiber opens up when its geometrical properties (characterized by the two angles $\phi$ and $\alpha$ ) are changed for the purpose of, say, transcription. As long as the positions of the nucleosomes at the DNA are fixed (as assumed here) $\phi$ and $b$ are always constant and hence it is the angle $\alpha$ with which the fiber geometry, i.e., its degree of swelling, is controlled. High accessibility means that the fiber opens up strongly with an increasing value of $\alpha$, leading to a strong reduction of the nucleosome line density. Hence our measure for accessibility, as introduced in Ref. [8], is the quantity $-\partial \Lambda / \partial \alpha$.

The purpose of this paper is to study how the entry-exit angle of the DNA at the nucleoseomes can be controlled via electrostatics. It can been seen in the cryo EM studies [5] that the fibers open up and become therefore more accessible when the ionic strength is reduced and that this opening is directly linked to an increase in $\alpha$. It was suggested that via other mechanisms (for instance, the acetylation of so-called histone tails 10], as explained in more detail below) the angle $\alpha$ and therefore the degree of swelling can be changed for a given section of the fiber and that this constitutes a biochemical means to control the transcriptional activity of genes.

Whereas the x-ray studies of the core particle [1] allow a detailed knowledge of the wrapped part of DNA it does not give insight into the conformational properties of the entering and exiting strands since the core particles were constituted from 146bp DNA only. One has therefore to refer to the electron cryomicrographs. In these micrographs it can be seen clearly that $10 \mathrm{~nm}$ stretches of the entering and exiting DNA strands are glued together forming a unique "stem motif" [0] ( $c f$. also Fig. 1(a)). The glueing of the two equally charged chains 


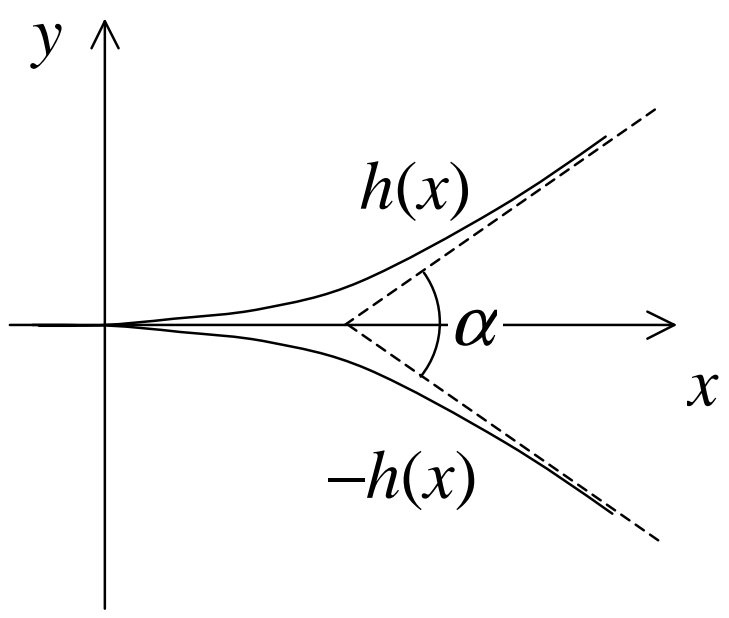

Fig. 2 - Idealized model for the entry-exit region of the DNA at the nucleosome. $h(x)$ and $-h(x)$ describe the conformations of the entering and exiting DNA sections, respectively. The asymptotic slope at large $x$ determines the entry-exit angle $\alpha$ and, in turn, the overall conformation of the chromatin fiber (as depicted in Fig. 1(a)).

is accomplished - amongst other things - via a special cationic protein, the so-called linker histone $\mathrm{H} 1$ as shown schematically in Fig. 1(b).

At physiological concentrations (roughly $100 \mathrm{mM}$ salt corresponding to a screening length of about $10 \AA$ ) the electrostatics is essentially short-ranged (note that the diameter of DNA is $20 \AA$ ). It seems therefore reasonable to assume that $\alpha$ is set within the small region where the two linker DNA are in close contact, i.e., in the stem region [11. This value of $\alpha$ in turn controls the large-scale secondary structure of chromatin, the $30 \mathrm{~nm}$-fiber. To mimic this situation we assume in the following two parallel DNA strands that are hold together tightly at $y=0$ for $x \leq 0$ and that are free for $x>0$, cf. Fig. 2. Because of their mutual electrostatic repulsion the two strands bend away from each other. When the two strands are far enough from each other their interaction is screened so that they asymptotically approach straight lines (neglecting thermal fluctuations as it is appropriate for the length scales under consideration). The two asymptotes define the opening angle $\alpha$ as indicated in Fig. 2. It is the purpose of this study to determine this angle and its dependence on different physical parameters [12].

We describe the conformation of the upper DNA chain by the height function $h(x)$. By symmetry the position of the lower strand is then given by $-h(x)$. From the above given considerations follow two boundary conditions at $x=0$ :

$$
h(0)=h^{\prime}(0)=0
$$

The entry-exit angle $\alpha$ is related to the slope of $h(x)$ at infinity as follows:

$$
\tan (\alpha / 2)=h^{\prime}(\infty)
$$

We model the two DNA chains as semiflexible polymers with persistence length $l_{P}$ and linecharge density $\lambda$ immersed in a salt solution characterized by the Bjerrum length $l_{B} \equiv e^{2} / \epsilon k_{B} T$ and the Debye screening length $\kappa^{-1}=\left(8 \pi c_{s} l_{B}\right)^{-1 / 2}(\epsilon$ dielectric constant of the solvent, $T$ temperature, $k_{B}$ Boltzmann constant and $c_{s}$ salt concentration). We assume that the charges 
on the DNA strands interact via a screened electrostatic potential. The free energy of the system is then given by

$$
\frac{F}{k_{B} T} \simeq \int_{0}^{\infty} d x\left[l_{P}\left(\frac{d^{2} h}{d x^{2}}\right)^{2}+2 l_{B} \lambda^{2} K_{0}(2 \kappa h(x))\right]
$$

The first term in the integral accounts for the bending of the two DNA strands and the second one describes the interaction between the two chains $\left(K_{0}(x)\right.$ being the $0 t h$ order modified Bessel function). Here the interaction of a given charge on one chain with all the charges on the other chain is approximated by the interaction of this charge with a straight chain at the distance $2 h$. The integral, eq. 3, is a good approximation as long as the value of $\alpha$ that follows from its minimization is sufficiently small, i.e., as long as $h^{\prime}(x) \ll 1$ for all $x$. We furthermore neglect here the interaction between charges on the same chain. The conformation of the upper chain, $h(x)$, is then the solution of the corresponding Euler-Lagrange equation (using $\left.K_{0}^{\prime}(x)=-K_{1}(x)\right)$

$$
l_{P} \frac{d^{4} h}{d x^{4}}-2 l_{B} \lambda^{2} \kappa K_{1}(2 \kappa h)=0
$$

together with four boundary conditions: Two are given at the origin, eq. 1, and two follow from the condition of straight "linkers" at infinity:

$$
h^{\prime \prime}(\infty)=h^{\prime \prime \prime}(\infty)=0
$$

Defining $\tilde{h}=2 \kappa h$ and introducing the dimensionless quantity $\tilde{x}=\left(4 l_{B} \lambda^{2} \kappa^{2} l_{P}^{-1}\right)^{1 / 4} x$, eq. 1 can be rewritten as

$$
\frac{d^{4} \tilde{h}}{d \tilde{x}^{4}}-K_{1}(\tilde{h})=0
$$

We denote the dimensionless asymptotic slope by $c_{0}=d \tilde{h} /\left.d \tilde{x}\right|_{\tilde{x}=\infty}$. It follows immediately that $\tan (\alpha / 2)$ is given by

$$
\tan (\alpha / 2)=h^{\prime}(\infty)=\frac{c_{0}}{\sqrt{2}}\left(\frac{l_{B}}{l_{P}}\right)^{1 / 4}\left(\frac{\lambda}{\kappa}\right)^{1 / 2}
$$

Equation 6 can be solved asymptotically for small value of $\tilde{h}$ where $K_{1}(\tilde{h}) \simeq 1 / \tilde{h}$. In leading order the asymptotic solution is given by $\tilde{h}(\tilde{x}) \simeq \tilde{h}_{0}(\tilde{x})=\tilde{x}^{2} \sqrt{(-\ln \tilde{x})}$. This solution fulfills the boundary conditions at the origin, eq. 11. The boundary conditions at infinity, eq. 5, are only marginally affected by the solution for small values of $\tilde{x}$ as can be seen by adding terms like $a \tilde{x}^{2}$ and $b \tilde{x}^{3}$. We can use this approximate solution to give a rough estimate for $c_{0}$. $\tilde{h}_{0}(\tilde{x})$ reaches its maximal slope 0.56 at $\tilde{x} \simeq 0.41$. For larger values of $\tilde{x}$ the approximate solution $\tilde{h}_{0}(\tilde{x})$ becomes more and more unreliable. This asymptotic analysis indicates that $c_{0}$ is of the order one 14. The asymptotic solution allows to estimate the free energy of the configuration since most of the bending and electrostatic contributions are localized close to the origin. Replacing $h(x)$ in eq. 3 by $h_{0}(x)=(\gamma x)^{2} \sqrt{-\ln (\gamma x)} / 2 \kappa$ with $\gamma=\left(4 l_{B} \lambda^{2} \kappa^{2} / l_{P}\right)^{1 / 4}$ and integrating from $x=0$ to $x \approx \gamma^{-1}$ (neglecting logarithmic contributions) we find that both, the bending contribution and the electrostatic contribution scale as $F / k_{B} T \approx l_{B}^{3 / 4} \lambda^{3 / 2} l_{P}^{1 / 4} \kappa^{-1 / 2}$.

Our calculation does not account for intramolecular electrostatic contributions. These can be included by adding the electrostatic persistence length $l_{O S F}=l_{B} \lambda^{2} / 4 \kappa^{2}$ (the OdijkSkolnick-Fixman length, cf. Ref. [13) to the bare persistence length, i.e., by replacing $l_{P}$ 
by $l_{P}+l_{O S F}$. This leads to $\tan (\alpha / 2) \propto\left(l_{O S F} /\left(l_{P}+l_{O S F}\right)\right)^{1 / 4}$. Note that for small salt concentrations (large $\left.\kappa^{-1}\right) l_{O S F}$ becomes increasingly important so that the angle approaches a universal value independent of $\kappa^{-1}, \lambda$ and $l_{B}$ - at least as long as the above stated approximations are still applicable in this limit. For the salt concentrations under consideration $l_{O S F} \ll l_{P}$ so that we disregard intramolecular contributions in the following.

We apply now our results to the problem of how the geometry of the chromatin fiber is controlled in vitro and, on a more tentative level, in vivo. The in vitro-experiments show that chromatin fibers "open up" with a decreasing salt concentration. From the electron cryomicrographs it was found that $\alpha \approx 85^{\circ}$ for $c_{s}=5 \mathrm{mM}$ and $\alpha \approx 45^{\circ}$ for $c_{s}=15 \mathrm{mM}$ \|5. Furthermore, from electron cryotomography it was estimated that $\alpha \approx 35^{\circ}$ for $c_{s}=80 \mathrm{mM}$ [5]. We expect from eq. 7 that $\alpha \simeq 2 \arctan \left(C c_{s}^{-1 / 4}\right)$ with $C$ being a constant. Let us take the angle at the highest salt concentration, $c_{s}=80 \mathrm{mM}$, as the reference value. From this follows $C=0.94$. With this value of $C$ we predict $\alpha \approx 51^{\circ}$ for $c_{s}=15 \mathrm{mM}$ and $\alpha \approx 64^{\circ}$ for $c_{s}=80 \mathrm{mM}$. Whereas the predicted value of $\alpha$ at the intermediate ionic strength is close to the experimental one, the value for low salt concentrations is noticably too low. However, we expect the theory to break down for such large values of $\alpha$.

How can the degree of swelling of the chromatin fiber be controlled in vivo? Under the assumption that the above mentioned geometry is valid the only parameter that might be under biochemical control is the linear charge density $\lambda$. It is known that there are flexible cationic tails, namely the C-tail of the linker histone as well as the lysine-rich N-tails of the core histones involved in determining the degree of swelling. In Fig. 1(b) we give a tentative picture of the conformation of two N-tails that protrude from the histone core (we assume here that these are the tails of the two so-called H3 core histones that are located close to the entry-exit point). It is known that if either of these components is missing the fiber does not fold appropriately (cf. Ref. [10] and references therein). As indicated in the Figure the tails might form a complex with the entering and exiting linker DNA in such a way that they effectively reduce its linear charge density $\lambda$. It is known that transcriptionally active regions in chromatin show an acetylation of the core histone tails (i.e., the cationic groups of the lysines are neutralized). In our tentative picture this acetylation mechanism would increase $\lambda$ and according to eq. 7 this would lead to an opening of the entry-exit angle $\alpha$. The acetylation might therefore be the first step in the decondensation of a stretch of the chromatin fiber that needs to be accessed for transcription [15].

Further steps might then involve the loss of the linker histones leading to $\alpha \approx \pi$, i.e., a beadon-string filament. After the loss of the linker histones the nucleosomes might even become mobile as it was reported in Ref. [19]. We recently suggested that this "nucleosome sliding" results from DNA "reptating" around the histone core with the help of intranucleosomal loops [20]. Loop formation might also be crucial for the actual transcription itself: It was suggested that RNA polymerase might elongate through the nucleosome by passing it in a loop [21. However, up to now a detailed knowledge of how transcription through chromatin is possible is still unclear and a matter of current research.

$$
* * *
$$

I wish to thank R. Bruinsma, A. Johner and G. Migliorini for useful discussions.

\section{REFERENCES}


[1] Luger K., Mäder A. W., Richmond R. K., Sargent D. F. and T. J. Richmond, Nature, 389 (1997) 251.

[2] Woodcock C. L. and Dimitrov S., Curr. Opim. Genet. Dev., 11 (2001) 130.

[3] Finch J. T. and Klug A., Proc. Natl. Acad. Sci. USA, 73 (1976) 1897.

[4] Woodcock C. L., Grigoryev S. A., Horowitz R. A., and Whitaker N., Proc. Natl. Acad. Sci. USA, 90 (1993) 9021.

[5] Bednar J., Horowitz R. A., Grigoryev S. A., Carruthers L. M., Hansen J. C., Koster A. J. and Woodcock C. L., Proc. Natl. Acad. Sci. USA, 95 (1998) 14173.

[6] Cui Y. and Bustamante C., Proc. Natl. Acad. Sci. USA, 97 (2000) 127.

[7] Katritch V., Bustamante C. and Olson W. K., J. Mol. Biol., 295 (2000) 29.

[8] Schiessel H., Gelbart W. M. and Bruinsma R., Biophys. J., 80 (2001) 1940.

[9] Widom J., Proc. Natl. Acad. Sci. USA, 89 (1992) 1095.

[10] van Holde K. and Zlatanova J., Proc. Natl. Acad. Sci. USA, 93 (1996) 10548.

[11] We assume here that internucleosomal interaction can be neglected. There is evidence from the stretching experiments [6] that the nucleosomes are "in contact" at a physiological ionic strength and that there is a short-range attraction between them. With the swelling of the fiber at lower ionic strength the internucleosomal interaction becomes less important and might be negligible (cf. our discussion of the internucleosomal interaction in Ref. [8]).

[12] A similar problem is that of a charged chain that is deflected by a rod perpendicular to it. This mimics a situation occuring in semidilute polyelectrolyte solutions and is discussed in Ref. 13], p 39.

[13] Barrat J.-L. and Johnny J.-F., Adv. Chem. Phys., 94 (1996) 1.

[14] Note that our analysis is based on the assumption of a vanishing distance between the two strands for $x \leq 0$. This leads to a logarithmic singularity of the curvature at $x=0$, i.e., to an infinite torque at the origin. The structure could only be stabilized for an infinite adsorption energy per length between the two strands for $x \leq 0$. A more realistic case is $\tilde{h}(x) \equiv \varepsilon \ll$ 0 for $x \leq 0$. In this case the following approximate solution can be constructed: $\tilde{x}^{4} / 24 \varepsilon-$ $\tilde{x}^{3} /\left(3 \tilde{x}_{0} \sqrt{-\ln \tilde{x}_{0}}\right)+\tilde{x}^{2} \sqrt{\left(-\ln \tilde{x}_{0}\right)}+\varepsilon$ for $\tilde{x}<\tilde{x}_{0}$ and $\tilde{h}_{0}(x)+\varepsilon$ for $\tilde{x}_{0}<\tilde{x} \ll 1$; here $\tilde{x}_{0}$ is the solution of $\tilde{x}_{0}^{2} \sqrt{-\ln \tilde{x}_{0}}=\varepsilon$. These two functions cross over smoothly at $\tilde{x} \approx \tilde{x}_{0}$ and obey Eq. 6 for $\tilde{x} \ll \tilde{x}_{0}$ and for $\tilde{x} \gg \tilde{x}_{0}$, respectively. This indicates that the solution is only marginally affected by the value of $\varepsilon$ as long as $\varepsilon \ll 0$ and that our original assumption, Eq. 1, is reasonable.

[15] The processes that are involved in the acetylation and deacetylation might be quite specific and involved as, for instance, discussed in Ref. 16. The histone tail modifications might serve specific functions via the modification of their secondary structure that in turn modifies their interaction with certain proteins 17. Recently there are even attempts to decipher a specific "language" of covalent histone modifications [18]. It might be that such specific processes act in concert with the more basic charge neutralization principle discussed here.

[16] Grunstein M., Nature, 389 (1997) 349.

[17] Hansen J. C., Tse C. and Wolffe A. P., Biochemistry, 37 (1998) 17637.

[18] Strahl B. D. and Allis D., Nature, 403 (2000) 41.

[19] Pennings S., Meersseman G. and Bradbury E. M., Proc. Natl. Acad. Sci. USA, 91 (1994) 10275.

[20] Schiessel H., Widom J., Bruinsma R. F. and Gelbart W. M., Phys. Rev. Lett., 86 (2001) 4414.

[21] Studitsky V. M., Clark D. J. and Felsenfeld G., Cell, 71 (1992) 371. 